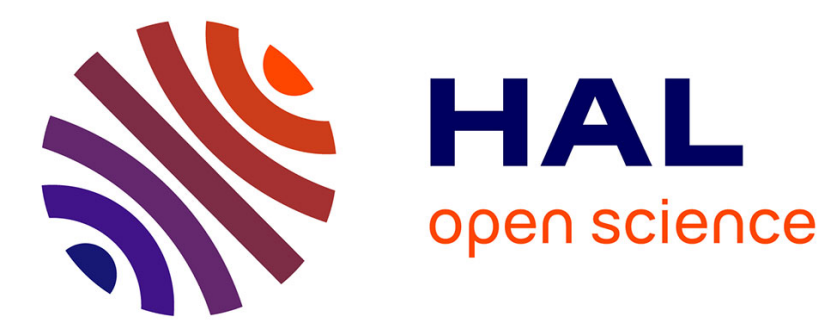

\title{
Comparison of microphone array denoising techniques and application to flight test measurements
}

Alice Dinsenmeyer, Quentin Leclere, Jérôme Antoni, Emmanuel Julliard

\section{To cite this version:}

Alice Dinsenmeyer, Quentin Leclere, Jérôme Antoni, Emmanuel Julliard. Comparison of microphone array denoising techniques and application to flight test measurements. 2019 AIAA/CEAS Aeroacoustics Conference, May 2019, Delft, Netherlands. hal-02147821

\section{HAL Id: hal-02147821 \\ https://hal.science/hal-02147821}

Submitted on 5 Jun 2019

HAL is a multi-disciplinary open access archive for the deposit and dissemination of scientific research documents, whether they are published or not. The documents may come from teaching and research institutions in France or abroad, or from public or private research centers.
L'archive ouverte pluridisciplinaire HAL, est destinée au dépôt et à la diffusion de documents scientifiques de niveau recherche, publiés ou non, émanant des établissements d'enseignement et de recherche français ou étrangers, des laboratoires publics ou privés. 


\title{
Comparison of microphone array denoising techniques and application to flight test measurements
}

\author{
Alice Dinsenmeyer* \\ INSA-Lyon, 69621 Villeurbanne, France \\ École Centrale de Lyon, 69134, Écully, France \\ Quentin Leclère $^{\dagger}$ \\ INSA-Lyon, 69621 Villeurbanne, France \\ Jérôme Antoni \\ INSA-Lyon, 69621 Villeurbanne, France \\ Emmanuel Julliard ${ }^{\S}$ \\ Airbus Operation S.A.S., 31060 Toulouse, France
}

This paper deals with the denoising of microphone array measurements. In many situations, flush mounted microphone arrays are polluted by a turbulent boundary layer, this is typically the case considering wind tunnels or inflight tests. Acoustic imaging results are strongly affected by this noise, classical approaches to solve this issue consist in removing the diagonal terms from the measured cross spectral matrix, or to implement background noise subtraction strategies. This can be sufficient for conventional beamforming approaches, but can be a limitation when implementing more advanced identification methods. This work introduces two alternative techniques, a first one based on a statistical model whose parameters are inferred from measurements (PFA - Probabilistic Factor Analysis), and a second one based on the use of noise-free references. The former is an original contribution of the work, while the later is a well known approach yet not often used in the present context. Both methods, as well as more classical approaches, are compared in the frame of inflight array measurements for the characterization of jet noise. It is shown that the proposed advanced denoising approaches show enhanced performances as compared to classical approaches when applying either conventional beamforming or inverse source characterization.

\section{Nomenclature}

Acronyms
BBSAN $=$ Broadband shock-associated noise
CSM $=$ Cross-Spectral Matrix
PCA $=$ Principal Component Analysis
PDF $=$ Probability Density Function
PFA $=$ Probabilistic Factor Analysis
SNR $=$ Signal-to-Noise Ratio
TBL $=$ Turbulent boundary layer

Variable names

$\boldsymbol{a}_{x}, \boldsymbol{b}_{x}=$ Parameters for the PDF of a variable $x$

$c \quad=$ Vector of PFA factors

$\boldsymbol{H}_{i} \quad=$ Steering vector for the $i^{t h}$ focusing point
$\boldsymbol{I}=$ Identity matrix

$I=$ Number of samples for the Gibbs sampler

$\boldsymbol{L} \quad=$ PFA mixing matrix

$M \quad=$ Number of microphones

$n=$ Noise vector

$N_{s} \quad=$ Number of snapshots

$\boldsymbol{S}_{y x}=$ Cross-spectral matrix of vectors $\boldsymbol{y}$ and $\boldsymbol{x}$

$\boldsymbol{y} \quad=$ Vector of measured data

$\boldsymbol{\alpha}=$ PFA factor weights

$\beta_{p} \quad=$ Noise relative intensity of the $p^{\text {th }}$ measurement

$\gamma^{2}=$ Variance of the PFA factors

$\kappa=$ Number of PFA factors

$\sigma^{2} \quad=$ Noise variance

\footnotetext{
*Ph.D. Student, Laboratoire Vibrations Acoustique \& Laboratoire de Mécanique des Fluides et d'Acoustique, alice.dinsenmeyer@insa-lyon.fr

$\dagger$ Assistant Professor, Laboratoire Vibrations Acoustique, quentin.leclere@insa-lyon.fr

†Professor, Laboratoire Vibrations Acoustique, jerome.antoni@insa-lyon.fr

$\S$ Acoustic engineer, Airbus acoustic department, emmanuel.julliard@airbus.com
} 


$$
\begin{array}{ll} 
& \text { Indices } \\
j & =\text { Snapshot index } \\
p & =p^{t h} \text { measurement configuration }
\end{array}
$$

\begin{tabular}{ll}
\multicolumn{3}{c}{ Mathematical notations } \\
$\mathcal{N}_{\mathbb{C}} \quad=$ Complex Gaussian PDF \\
$\mathcal{E} \quad=$ Exponential PDF \\
$\mathcal{I} G \quad=$ Inverse-Gamma PDF
\end{tabular}

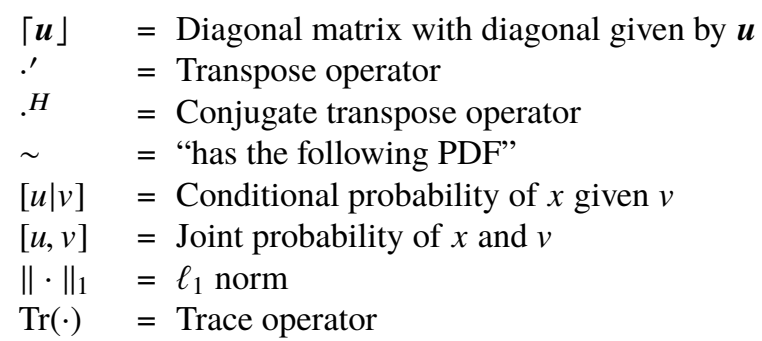

\section{Introduction}

$\mathrm{M}$ Icrophone array measurements are often polluted by extraneous noise that can be ambient, electronic or induced by a turbulent boundary layer, and it can strongly affect the quality of source characterization. Especially, if the microphones are located close or inside a flow, during wind tunnel or inflight tests for example, the signal-to-noise ratio (SNR) may become very poor and a denoising step is necessary.

In this context, multiple denoising processes have been developed, based on various strategies such as Principal Component Analysis [1], wavenumber filtering [2], diagonal optimization [3, 4] and some methods also make use of a background noise measurement [5-7]. Another common practice is to set the diagonal of the measured cross-spectral matrix (CSM) to zero. It is based on the fact that the turbulent boundary layer (TBL) noise often concentrate on the diagonal elements of the measured CSM, because its correlation length is smaller than the microphone inter-spacing, but doing so can lead to negative CSM and underestimation of the characterized sources.

In this paper, we introduce two other denoising techniques. The first denoising method is based on a statistical inference problem that exploits the properties of the TBL noise and the acoustical contribution. The second one makes use of noise-free reference signals. Then, an experimental section is dedicated to the application of these methods to inflight large aircraft tests and the influence of each denoising techniques is studied through a comparison of denoised autospectra and resulting acoustic imaging maps.

\section{Denoising using Factor analysis}

The main idea of this method is to exploit the difference in the statistical properties of the acoustic and the aerodynamic noise. The acoustical part is supposed to have a correlation length larger than the microphone interspacing. On the contrary, the TBL noise is supposed to be random, with a very short correlation length compared to microphone interspacing.

It has been shown that the rank of the acoustical CSM is directly related to the number of statistically independent sources [8]. In other words, the rank gives the number of uncorrelated component that are necessary to reconstruct the sound field. This number is supposed to be small compared to the number of microphones. Therefore, the acoustical CSM can be modeled with a low-rank matrix. Following the assumption of a small correlation length for the TBL noise, the estimates of the associated CSM tends to be a diagonal matrix, when the number of averages tends to infinity.

From these postulates, we propose to decompose the measured CSM using Probabilistic Factor Analysis (PFA). PFA is an inference problem that aims at fitting the measured data with a statistical model.

\section{Statistical model}

PFA models the measured pressure field as linear combinations of a small number of factors, which captures the acoustical part in our denoising case, plus an error term, which stands for the TBL noise part. It can be written as follow:

$$
\boldsymbol{y}_{j}=\boldsymbol{L} \boldsymbol{c}_{j}+\boldsymbol{n}_{j} \quad j=1, \ldots, N_{s},
$$

where $c$ is the vector of $\kappa<M$ latent factors, $\boldsymbol{L} \in \mathbb{C}^{M \times \kappa}$ is the matrix that mixes factors, $\boldsymbol{n}$ is a term of residual errors (independent from the factors), and index $j$ is the snapshot number. In the classical PFA formulation, the user has to select the number of factor $\kappa$, which gives the maximal rank of the acoustical CSM. We propose to extend this model to consider $\kappa$ as an unknown of the model, to be inferred. To do so, a diagonal matrix $\lceil\alpha\rfloor$ is added to weight the factors:

$$
\boldsymbol{y}_{j}=\boldsymbol{L}\lceil\alpha\rfloor \boldsymbol{c}_{j}+\boldsymbol{n}_{j} \quad j=1, \ldots, N_{s}
$$


This weighting matrix should cancel the smallest factors, and thus allows an automatic selection of the model order by setting the optimal number of factors to zero.

In the context of factor analysis, all the unknown parameters of this model are seen as random variables, with the following assigned probability density functions (PDF), called priors:

$$
\begin{aligned}
\boldsymbol{c} & \sim \mathcal{N}_{\mathbb{C}}\left(0,\left\lceil\boldsymbol{\gamma}^{2}\right\rfloor\right), \\
\boldsymbol{n} & \sim \mathcal{N}_{\mathbb{C}}\left(0,\left\lceil\boldsymbol{\sigma}^{2}\right\rfloor\right), \\
\boldsymbol{\alpha} & \sim \mathcal{E}\left(\boldsymbol{a}_{\alpha}\right), \\
\boldsymbol{L} & \sim \mathcal{N}_{\mathbb{C}}\left(0, \frac{\boldsymbol{I}}{\kappa}\right),
\end{aligned}
$$

where $\mathcal{E}$ is the exponential PDF and $\mathcal{N}_{\mathbb{C}}$ is the complex Gaussian PDF. $\boldsymbol{\gamma}^{2}, \boldsymbol{\sigma}^{2}$ and $\boldsymbol{a}_{\alpha}$ are hyperparameters of the model and $\boldsymbol{I}$ stands for the identity matrix.

\section{Optimization step}

Rather than fitting the measured pressures to model 11, the inference problem is solved using the measured CSM $\boldsymbol{S}_{y y}=N_{s}^{-1} \sum_{j} \boldsymbol{y}_{j} \boldsymbol{y}_{j}^{H}\left({ }^{H}\right.$ is the Hermitian operator). Therefore, the model 1 can be approximated (ignoring the cross-terms) by:

$$
\boldsymbol{S}_{y y}=\boldsymbol{L}\lceil\alpha\rfloor \boldsymbol{S}_{c c}\lceil\alpha\rfloor \boldsymbol{L}^{H}+\boldsymbol{S}_{n n}
$$

Several methods exist to solve this inference problem. Here, a Bayesian approach is chosen and a Gibbs sampler (a Markov chain Monte Carlo algorithm) is used to approximate the unknown parameters by maximizing the posterior distribution, which reads:

$$
\hat{\boldsymbol{S}}_{c c}, \hat{\boldsymbol{L}}, \hat{\boldsymbol{\sigma}}^{2}, \hat{\boldsymbol{\alpha}}=\operatorname{argmax}\left[\boldsymbol{S}_{c c}, \boldsymbol{L}, \boldsymbol{\sigma}^{2}, \boldsymbol{\alpha} \mid \boldsymbol{y}\right],
$$

where the notation $[x]$ stands for the probability density of the random variable $x$. In such approach, the parameters of the prior distributions can also be inferred and they have their own priors, called hyperpriors. In the model of Eq. (2), factor and noise variances are unknown and they are classically sampled following inverse-gamma $(\mathcal{I} G)$ distributions:

$$
\begin{aligned}
\gamma^{2} & \sim \mathcal{I} \mathcal{G}\left(\boldsymbol{a}_{\gamma}, \boldsymbol{b}_{\gamma}\right), \\
\boldsymbol{\sigma}_{n}^{2} & \sim \mathcal{I} \mathcal{G}\left(\boldsymbol{a}_{\sigma}, \boldsymbol{b}_{\sigma}\right) .
\end{aligned}
$$

Shape and scale parameters $\boldsymbol{a}$ and $\boldsymbol{b}$ can be set according to the prior knowledge of the noise and acoustic source variance. In the following, non-informative priors are used : dealing with poor SNR, mean value of $\gamma^{2}$ and $\sigma^{2}$ are set equal to the mean of the measured autospectra, and their variance is set arbitrarily 10 times higher. Concerning the slope parameter $\boldsymbol{a}_{\alpha}$ in Eq. (5), it is chosen with an exponential decay on the factors with no normalization needed, since the solution for $\boldsymbol{L}, \boldsymbol{c}$ and $\boldsymbol{\alpha}$ is not unique (but their product is). Finally, the initialization for $\boldsymbol{L}$ is random.

Note that this method is closely related to principal component analysis (PCA), previously used by [9] and [10] to reduce TBL noise. Similarly to PCA, the data are linearly decomposed in a reduced set of variables that leads to a reduction of the data dimension. In the PCA formulation, these variables are orthogonal, which is not necessarily the case for PFA. Therefore, PFA can better take into account assumptions about the latent structures of the data. Especially, Gibbs sampler being very flexible, a correlation structure of the hydrodynamic noise could be taken into account in the inference model. Moreover, PFA provides a non-negative decomposition of the acoustical CSM that can be used to reduce the dimension of an imaging process.

\section{Use of background noise and multiple measurements}

PFA can be used to denoise a single CSM, but in the case where more data are available, it can be useful to exploit all of them to improve the accuracy of the inferred parameters. Indeed, during the flight tests, measurements are performed for several engine speeds and a measurement at idle speed is used as a background TBL noise reference. The statistical properties of the TBL noise is supposed to be unchanged from one working point $p$ to another. Therefore, the inferred 
noise variance $\sigma^{2}$ is common to all datasets (varying by a multiplicative factor $\beta_{p}$ ) but a different acoustical part is inferred for each dataset. The model reads:

$$
\boldsymbol{y}_{j_{p}}=\left\{\begin{array}{cc}
\boldsymbol{n}_{j_{p}} & \text { for } p=1, \text { the idle working point } \\
\boldsymbol{L}_{p}\lceil\alpha\rfloor_{p} \boldsymbol{c}_{j_{p}}+\boldsymbol{n}_{j_{p}} & \text { for } p=2, \ldots, P
\end{array}\right.
$$

with following priors:

$$
\begin{aligned}
\boldsymbol{c}_{p} & \sim \mathcal{N}_{\mathbb{C}}\left(0, \gamma_{p}^{2}\right), \\
\boldsymbol{n}_{p} & \sim \mathcal{N}_{\mathbb{C}}\left(0, \beta_{p}\left\lceil\sigma^{2}\right\rfloor\right), \\
\beta_{p} & \sim \mathcal{I} \mathcal{G}\left(a_{\beta}, b_{\beta}\right), \\
\boldsymbol{\alpha}_{p} & \sim \mathcal{E}\left(\boldsymbol{a}_{\alpha_{p}}\right), \\
\boldsymbol{L}_{p} & \sim \mathcal{N}_{\mathbb{C}}\left(0, \frac{\boldsymbol{I}}{\kappa}\right) .
\end{aligned}
$$

The scalar parameter $\beta_{p}$ allows the noise to vary in amplitude for each working point, but not in shape. The parameters with subscript $p$ are first drawn in the distributions conditioned to each working point data, and the noise variance $\sigma^{2}$ is then drawn knowing the whole data. The pseudocode of the Gibbs sampler that solves this problem is given by Algorithm 11 The optimal parameters are obtained after averaging the $I / 2$ last output samples.

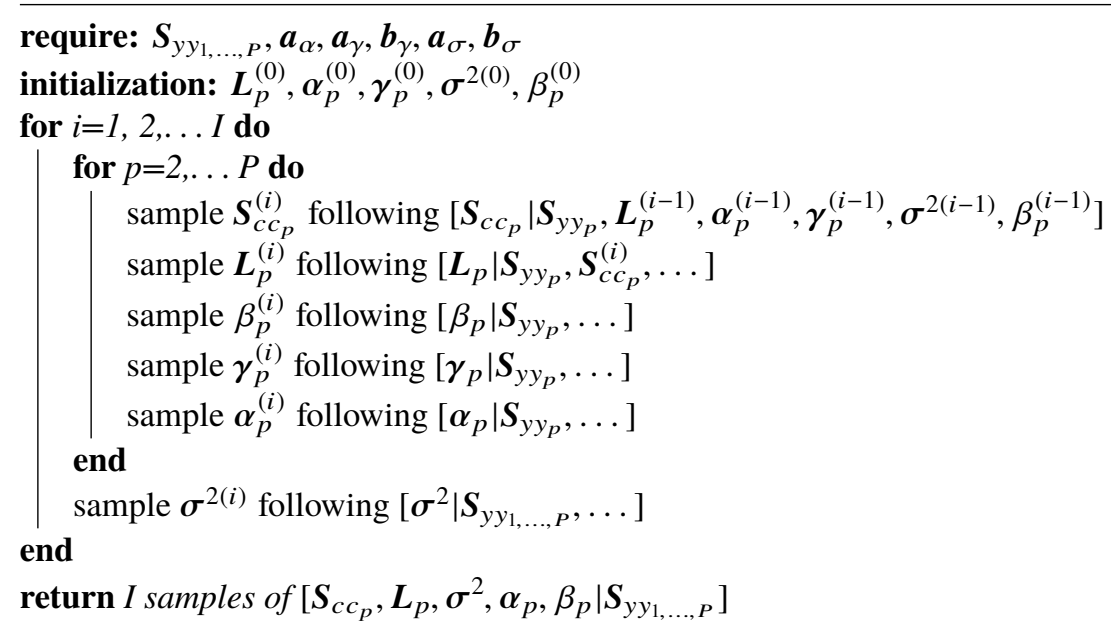

\section{Algorithm 1: Gibbs sampling for PFA denoising, extracting a common noise from multiple measurement datasets.}

\section{Denoising using reference signals}

The noise affecting fuselage microphones is mainly attributed to the contribution of the TBL. In cases where some noise-free reference signals are available, it is possible to apply referenced denoising techniques in order to clean-up microphone array signals. Let $x$ and $y$ stand for a set of reference and array output signals, respectively. The CSM of outputs conditioned by references is given by

$$
S_{y y}^{x}=S_{y x} S_{x x}^{-1} S_{x y}
$$

This formulation is accordance with Bendat and Piersol's Conditioned Spectral Analysis [11]: autospectra on the diagonal of $\boldsymbol{S}_{y y}^{x}$ are corresponding to multiple coherent output spectra, the multiple coherence being directly given by the ratio between diagonal terms of $\boldsymbol{S}_{y y}^{x}$ and $\boldsymbol{S}_{y y}$.

Note that this denoising approach assumes that the noise affecting the output signals is not contributing to references. References are not necessarily completely noise-free, however the noise affecting references has to be independent from the noise contributing to outputs.

Two major differences exist between this referenced denoising approach as compared to the PFA technique presented in the preceding section: 
- referenced denoising requires several additional sensors, recorded simultaneously with the microphone array signals,

- referenced denoising does not require any characterization of the background noise or multiple engine operating point.

However, it is still interesting to compare results of referenced and blind denoising methodologies, because they share the same purpose (denoising). Considering that hypothesis and required data are strongly different, a good agreement between their results can be considered as a cross validation of both approaches.

For the considered experimental application, reference signals are accelerometers and microphones positioned on the inner side of the fuselage and inside the aircraft cabin, respectively. Those sensors are used as references because they are supposed to be much less affected by the TBL than external microphones : the fuselage is known to act as a low-pass filter in the wavenumber domain, the SNR (acoustic part over TBL part) of inside sensors is thus expected to be much more favorable. On the other hand, inside microphones are also disturbed by interior noise sources, mainly the air conditioning system. However, this noise being independent from the TBL, this should not cause any issue.

\section{Experimental application: Large aircraft flight tests}

\section{Experimental setup}

Denoising methods presented in this paper are used to characterize acoustic sources emitted by an Airbus large aircraft in stationary flight. Flight conditions are very similar to classical cruise flights, at altitude of $35,000 \mathrm{ft}$ and flight Mach number 0.85. Microphone acquisition are performed synchronously, involving 34 microphones flushmounted on the aft portside fuselage, 9 internal microphones, located in the aft cabin and 6 accelerometers fixed on the internal side of the fuselage, on same side as the microphone array. The external array configuration is presented in Fig. 1. Here, the experimental setup is such that the main contribution comes from the engine jet noise. Measurements are performed at 11 different engine speeds, including idle, referred as background noise. Each record lasts 60 seconds and the CSM are calculated with a resolution of $4 \mathrm{~Hz}$ (Hanning window, $70 \%$ overlap).
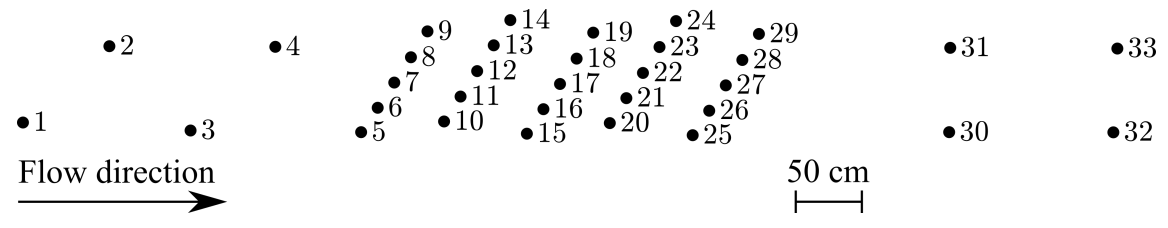

\section{Fig. 1 Geometry of the external microphone antenna}

\section{Denoised CSM}

Raw averaged autospectra obtained at a high engine speed are presented in Fig. 2 together with the background noise autospectra. Autospectra are also presented after subtracting the background noise CSM from the CSM of the considered operating point. This denoising method is referred as "Background Subtraction". Its underlying assumptions are that the noise remains statistically stationary during all the experiments and that the SNR is sufficiently high.

On the same figure are also plotted the mean autospectra obtained using referenced and PFA denoising. For the former, reference signals are the 6 fuselage accelerometers and the 9 inside microphones. Concerning the latter, the noise is extracted using all the 10 working data points (Algorithm 1 ran with $p=1, \ldots, 10$ ). From these spectra one can see that the average SNR is about $3 \mathrm{~dB}$, and it slightly increases in the frequency band between 1 and 3 . This spectra is dominated by the broadband shock-associated noise (BBSAN), generated by the dual-stream underexpanded supersonic jet [12]. In this frequency band, PFA and referenced denoising results are in agreement.

At higher frequencies, PFA does not reconstruct any coherent spectra. The SNR goes lower and there is not a sparse number of dominant sources anymore. Therefore, the PFA problem becomes poorly conditioned. In this Bayesian approach, the selection of priors can be seen as a regularization process, and the result can be sensitive to this prior selection in the case where the inference model diverges from reality.

On Fig. 3 are presented the same autospectra for each microphone, zoomed in the BBSAN frequency band. At very low frequency, the SNR is very poor and background subtraction gives inconsistent negative values. At these frequencies, TBL is very correlated and PFA fails to denoise : the assumption of uncorrelated noise does not hold true 


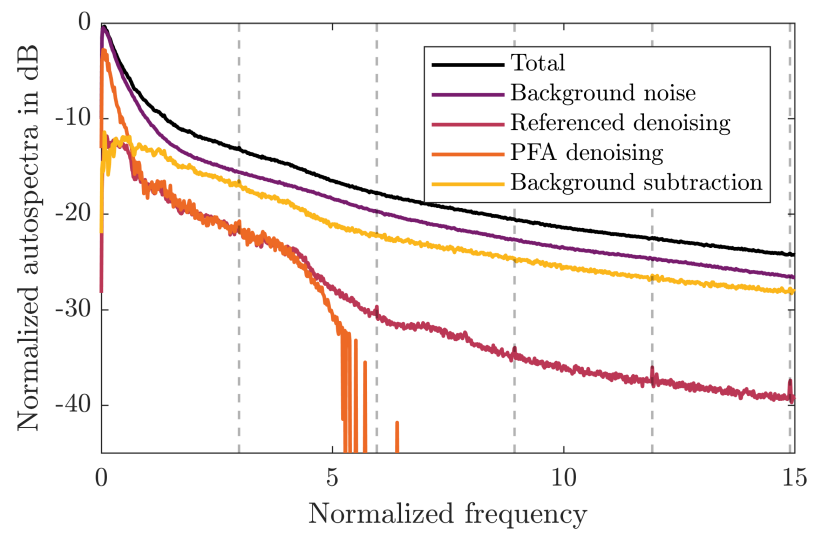

Fig. 2 Wideband autospectra averaged over the external microphones, raw ( - ), background noise ( - ), after referenced denoising ( $\_$), after PFA denoising ( - ), background subtraction $(-)$). Vertical lines $(---)$ are located at the blade passing frequencies.

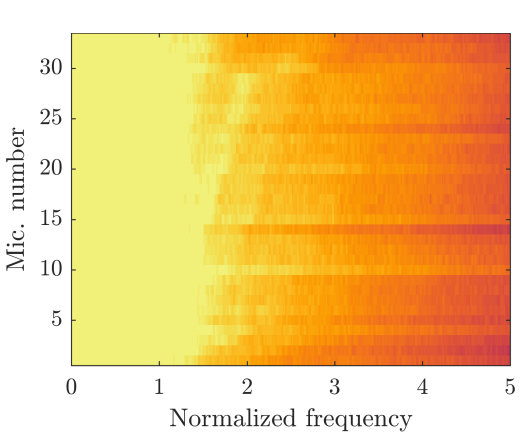

(a) Raw (high engine speed).

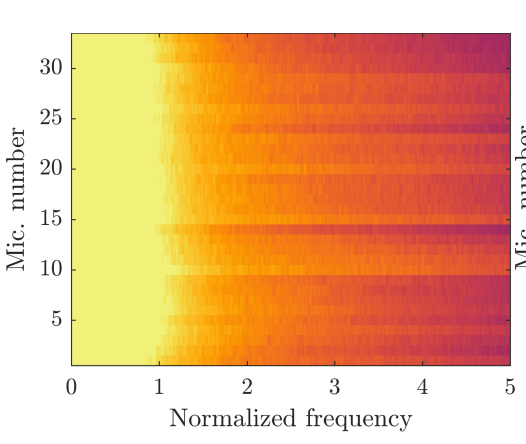

(b) Background noise (idle engine speed).

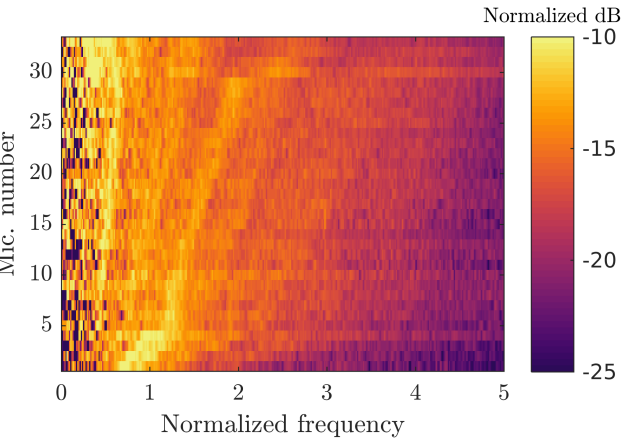

(c) Background subtraction.

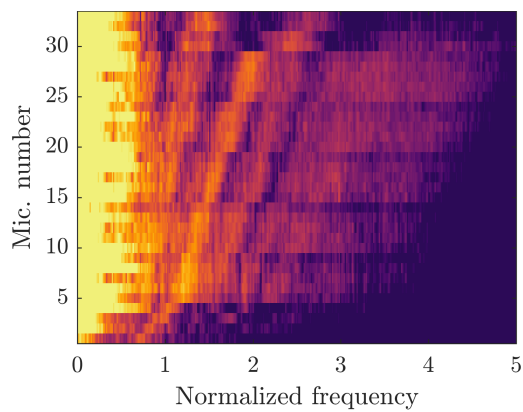

(d) PFA denoising.

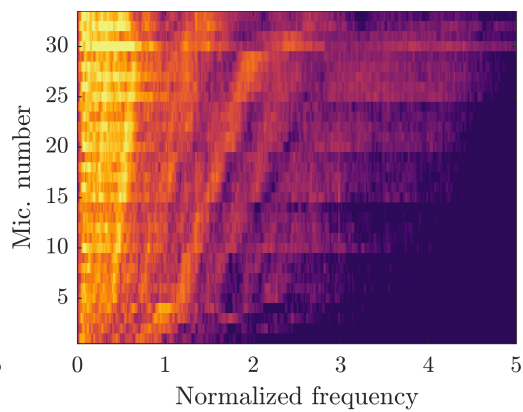

(e) Referenced denoising.

Fig. 3 Autospectra zoomed in the BBSAN frequency band, with no denoising (a), obtained at idle engine speed (b), after background subtraction (c), after PFA denoising (d) and after reference based denoising (e), Colorscale is the same for all graphs and it is clipped for better comparison.

and thus, the correlated noise is identified as a part of the acoustical CSM. In the range of frequency 1 to 3 , coherent interactions of shock cells leads to directivity patterns that clearly appear after denoising. Harper-Bourne and Fisher [13] proposed to model this BBSAN by regularly spaced partially coherent monopoles, which should induce interference patterns in the far field. 


\section{Acoustic imaging results}

In this section, denoised acoustic data are post-processed for acoustic imaging purpose. The study is conducted in a frequency range where the BBSAN is supposed to dominate. Equivalent sources are distributed along the jet axis, starting from the engine bypass outlet position up to this position plus 16 times the nozzle exit diameter. The acoustic propagation between the equivalent sources and the microphone array is supposed to be affected by a uniform flow.

\section{Beamforming}

First of all, measurement data are processed using a conventional beamforming approach (similarly to some related literature data [14]). The beamforming coherence, indicating the correlation between the measured sound field and the sound field generated by a single monopole at the focusing point, is given by:

$$
C_{i}=\frac{\boldsymbol{H}_{i}^{\prime} \boldsymbol{S}_{y y} \boldsymbol{H}_{i}}{\left(\boldsymbol{H}_{i}^{\prime} \boldsymbol{H}_{i}\right) \operatorname{Tr}\left(\boldsymbol{S}_{y y}\right)}
$$

where the prime is the transpose operator and $\boldsymbol{H}_{i}$ is the steering vector containing convected Green functions between the $i^{\text {th }}$ focusing point and the microphones. Four different $\boldsymbol{S}_{y y}$ CSM are considered :

- the raw CSM,

- the CSM denoised by subtraction of the CSM of the background noise,

- the CSM denoised using the PFA blind approach,

- the CSM denoised using the referenced approach.

The beamforming coherence obtained for these 4 cases are drawn in Fig. 4 . All the maps are exhibiting a main lobe around the normalized position 6 . However, the level of this lobe shows significant differences for the 4 cases, with a maximum at about $-8,-5,-2$ and $-3 \mathrm{~dB}$, respectively to the list. It seems thus that the advanced denoising approaches (PFA and referenced) performs significantly better than the basic background noise subtraction.

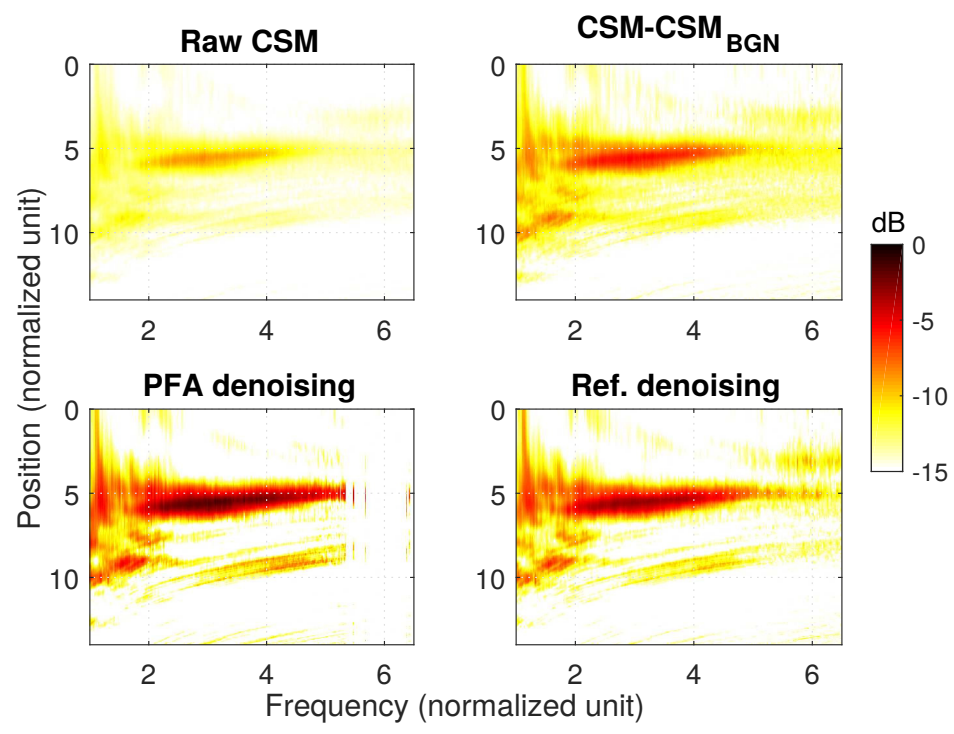

Fig. 4 Beamforming coherence (in $\mathrm{dB}$ ) as a function of focusing point and frequency using the raw CSM (up left), background noise subtraction (up right), CSM using PFA denoising (bottom left) and reference based denoising (bottom right).

\section{Bayesian focusing}

The 4 different CSM are also used as input for a more advanced imaging approach, Bayesian focusing, which is an inverse method based on an Iterative Reweighted Least Squares (IRLS) algorithm and a Bayesian regularization [15-17]. An interest of this method is that the user can define a sparsity constraint, between 2 (no sparsity) and 0 (strong sparsity). 
The source of interest here is the BBSAN, which is often assumed in the literature to behave as a set of discrete partially coherent monopoles. A strong sparsity is thus assumed and the sparsity parameter is set to 0 . Results are drawn in Fig. 5, for the 4 cases.

The Bayesian regularization selects automatically the regularization parameter depending on the inferred estimation of the signal to noise ratio. Results are thus expected to be affected by the denoising quality. For instance, looking more closely to the source distributions for a normalized position between 4 and 6, the raw CSM based results have only one source, while a second source appears at some frequency bins when subtracting the background noise CSM. Considering the denoised cases (PFA and referenced), two distinct sources are clearly identified.

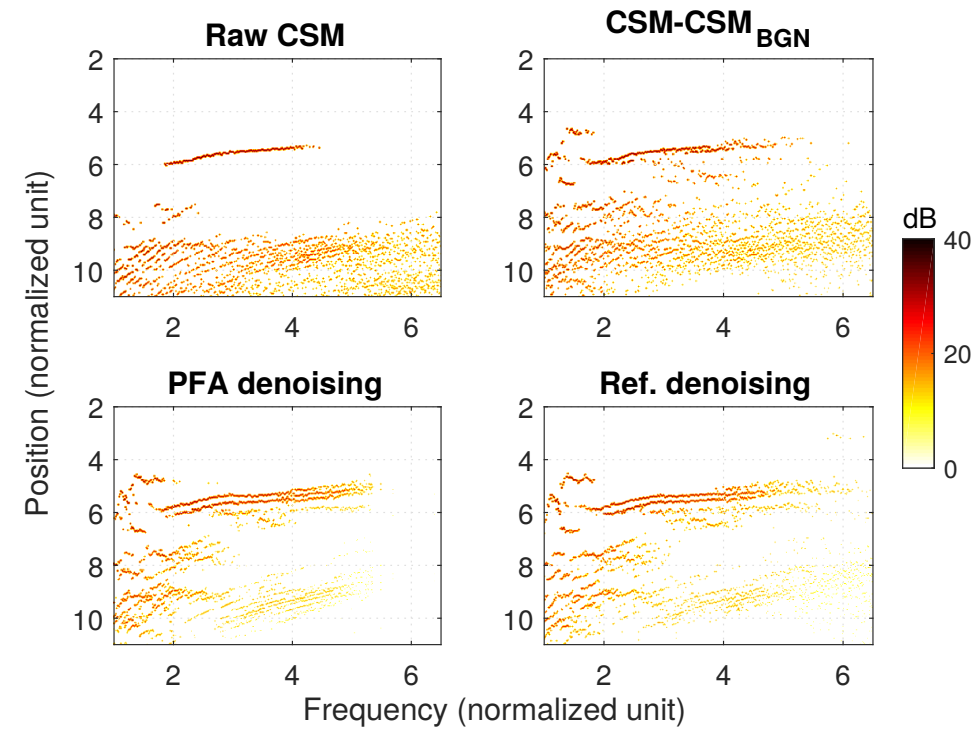

Fig. 5 Identified source powers (in dB) as a function of source position and frequency using the raw CSM (up left), background noise subtraction (up right), CSM using PFA denoising (bottom left) and reference based denoising (bottom right).

\section{Reconstruction error}

It is not easy to objectively compare identification results, considering that the true source distribution is not known. However, the performance of the different approaches can be indirectly assessed by comparing the CSM used as input and the reconstructed CSM, using the identified source distribution and the propagation operator. The following error indicator is used to compare input and output CSM :

$$
E=\frac{\left\|\boldsymbol{S}_{y y}-\boldsymbol{H} \boldsymbol{S}_{q q} \boldsymbol{H}^{\prime}\right\|_{1}}{\left\|\boldsymbol{S}_{y y}\right\|_{1}+\left\|\boldsymbol{H} \boldsymbol{S}_{q q} \boldsymbol{H}^{\prime}\right\|_{1}},
$$

where $\|\cdot\|_{1}$ is the $\ell_{1}$ norm, $\boldsymbol{S}_{q q}$ is the source CSM, and $\boldsymbol{H}$ the matrix of convected Green's functions between sources and microphones. Note that the same formula can be used for beamforming, with $S_{q q}$ the autopower of the beamforming maximum, and $\boldsymbol{H}=\boldsymbol{H}_{\boldsymbol{i}}$ the Green's functions between the beamforming maximum location and the microphones. This indicator is drawn in Fig. 6 (right), for the 4 cases, and is compared to the same error indicator based on a single monopole sources located at the maximum of the beamforming map (left). Results based on the inverse method exhibit significantly lower error indicators as compared to the single monopole model, showing the interest of considering more complex source distributions. This indicator shows also significant differences, for the inverse method, between the 4 input CSM : the maximum error is obtained with the raw CSM, and slightly better results are observed with the subtraction of the background noise CSM. Advanced denoising techniques show much better results, the PFA method seems to perform better than the referenced approach in a wide frequency range, even if some issues are encountered with PFA in the highest frequencies. 

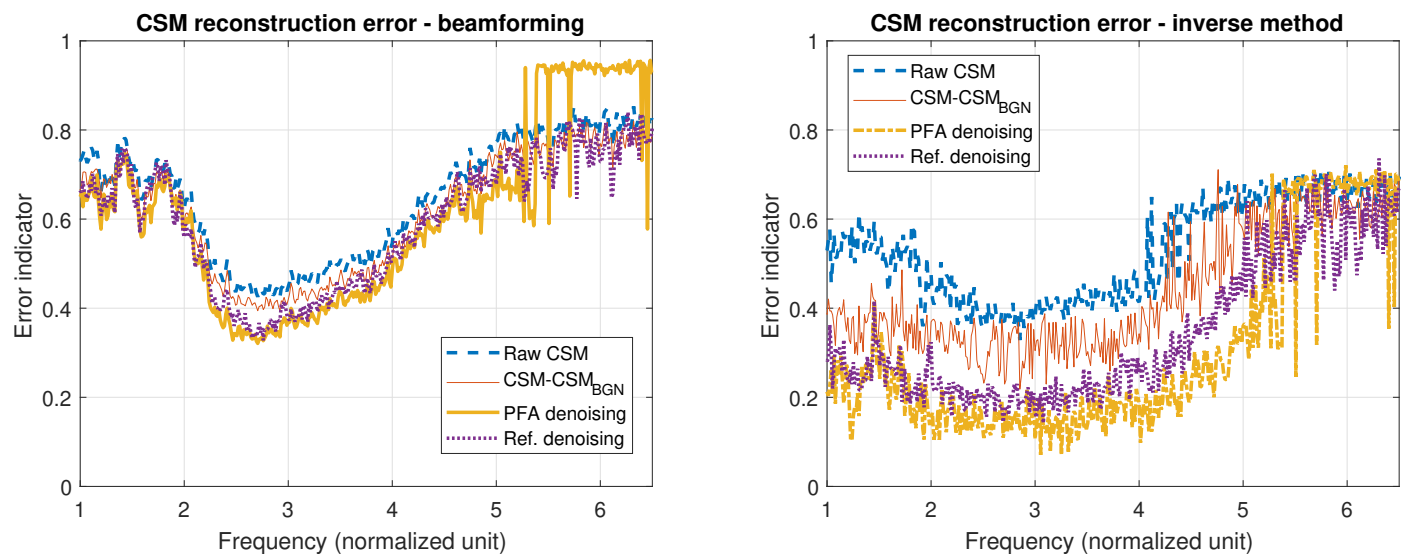

Fig. 6 Acoustic imaging : reconstructed CSM error indicator. Single monopole at the beamforming maximum location (left) and identification results (right).

\section{Conclusion}

The two proposed methods, PFA and referenced denoising, rely on very different hypothesis and data requirements, but the denoised spectra are very similar in the low and medium frequency band. They both provide promising TBL noise reduction and improve beamforming and inverse imaging as compared to the classical background subtraction, especially when considering the reconstruction error. At high frequencies, PFA method does not converge but multiple strategies could correct this problem, such as the use of a more robust sampler or an extension of the inference model.

\section{Acknowledgments}

This work was performed within the framework of the Labex CeLyA of the Universite de Lyon, within the programme 'Investissements d'Avenir' (ANR-10-LABX-0060/ANR-16-IDEX-0005) operated by the French National Research Agency (ANR). This work was also performed in the framework of Clean Sky 2 Joint Undertaking, European Union (EU), Horizon 2020, CS2-RIA, ADAPT project, Grant agreement no 754881.

\section{References}

[1] Druault, P., Hekmati, A., and Ricot, D., "Discrimination of acoustic and turbulent components from aeroacoustic wall pressure field," Journal of Sound and Vibration, Vol. 332, No. 26, 2013, pp. 7257 - 7278. doi:10.1016/j.jsv.2013.07.019.

[2] Arguillat, B., Ricot, D., Bailly, C., and Robert, G., "Measured wavenumber: Frequency spectrum associated with acoustic and aerodynamic wall pressure fluctuations," The Journal of the Acoustical Society of America, Vol. 128, No. 4, 2010, pp. 1647-1655. doi:10.1121/1.3478780.

[3] Dougherty, R., "Cross spectral matrix diagonal optimization,” 6th Berlin Beamforming Conference, 2016.

[4] Hald, J., "Removal of incoherent noise from an averaged cross-spectral matrix," The Journal of the Acoustical Society of America, Vol. 142, No. 2, 2017, pp. 846-854. doi:10.1121/1.4997923.

[5] Blacodon, D., "Spectral estimation noisy data using a reference noise," Proceedings on CD of the 3rd Berlin Beamforming Conference, 24-25 February, 2010, 2010.

[6] Boll, S., "Suppression of acoustic noise in speech using spectral subtraction," IEEE Transactions on acoustics, speech, and signal processing, Vol. 27, No. 2, 1979, pp. 113-120. doi:10.1109/tassp.1979.1163209.

[7] Bulté, J., "Acoustic array measurements in aerodynamic wind tunnels: a subspace approach for noise suppression," 13th AIAA/CEAS Aeroacoustics Conference (28th AIAA Aeroacoustics Conference), 2007, p. 3446. doi:10.2514/6.2007-3446.

[8] Yoon, S., and Nelson, P. A., "A method for the efficient construction of acoustic pressure cross-spectral matrices," Journal of sound and vibration, Vol. 233, No. 5, 2000, pp. 897-920. doi:10.1006/jsvi.1999.2888. 
[9] Finez, A., Pereira, A. A., and Leclère, Q., "Broadband mode decomposition of ducted fan noise using cross-spectral matrix denoising," Fan Noise 2015, Lyon, France, 2015.

[10] Amailland, S., Thomas, J.-H., Pézerat, C., and Boucheron, R., "Boundary layer noise subtraction in hydrodynamic tunnel using robust principal component analysis," The Journal of the Acoustical Society of America, Vol. 143, No. 4, 2018, pp. $2152-2163$. doi:10.1121/1.5023685.

[11] Bendat, J., and Piersol, A., Engineering applications of correlation and spectral analysis, Wiley-Interscience, New York, 1980.

[12] Tam, C. K., Pastouchenko, N. N., and Viswanathan, K., "Broadband shock-cell noise from dual stream jets," Journal of Sound and Vibration, Vol. 324, No. 3-5, 2009, pp. 861-891. doi:10.1016/j.jsv.2009.02.012.

[13] Harper-Bourne, M., and Fisher, M. J. F., "The noise from shock waves in supersonic jets-Noise Mechanism," Agard Conference on Noise Mechanisms, 1973.

[14] Huber, J., Britchford, K., Laurendeau, E., Fleury, V., Bulté, J., Sylla, A., and Long, D., "Understanding and reduction of cruise jet noise at model and full scale," 15th AIAA/CEAS Aeroacoustics Conference (30th AIAA Aeroacoustics Conference), 2009, p. 3382. doi:10.2514/6.2009-3382.

[15] Antoni, J., “A Bayesian approach to sound source reconstruction: Optimal basis, regularization, and focusing," Acoustical Society of America Journal, Vol. 131, 2012, p. 2873. doi:10.1121/1.3685484.

[16] Pereira, A., Antoni, J., and Leclère, Q., "Empirical Bayesian regularization of the inverse acoustic problem,” Applied Acoustics, Vol. 97, 2015, pp. 11 - 29. doi:10.1016/j.apacoust.2015.03.008.

[17] Leclère, Q., Pereira, A., Bailly, C., Antoni, J., and Picard, C., “A unified formalism for acoustic imaging based on microphone array measurements," International Journal of Aeroacoustics, Vol. 16, No. 4-5, 2017, pp. 431 - 456. doi:10.1177/1475472x17718883. 